ISSN: 2674-8584 V.1 - N.1 - 2020

\title{
EFEITOS DO MÉTODO PILATES EM PACIENTES COM DOR LOMBAR CRÔNICA - UMA REVISÃO
}

\section{EFFECTS OF THE PILATES METHOD IN PATIENTS WITH CHRONIC LOW BACK PAIN - A REVIEW}

\begin{abstract}
Mayure Silva Pego Acadêmica do $9^{\circ}$ período de Fisioterapia da Universidade Presidente Antônio Carlos UNIPAC. E-mail:mayurepego22@gmail.com
\end{abstract}

Oralda Gomes da Cunha Acadêmica do $9^{\circ}$ período de Fisioterapia da Universidade Presidente Antônio Carlos -

UNIPAC. E-mail: oraldasalao16@gmail.com

André Luiz Velano de Souza Especialista em Fisioterapia Esportiva Professor orientador. E-mail: andrevelanofisio@gmail.com

\section{Resumo}

A dor lombar é comumente definida como dor ou desconforto localizado na área do aspecto posterior do corpo, desde a margem inferior da décima segunda costela até as pregas glúteas inferiores, com ou sem dor referida um ou ambos os membros inferiores. A dor lombar crônica é caracterizada como uma condição clínica de dor que persiste por um período maior que três meses. O Método Pilates apresenta-se como um tratamento clínico que envolve um programa de treinamento físico e mental, que considera o corpo e a mente uma unidade, sendo assim podendo trazer uma melhora global na qualidade de vida de indivíduos que apresentam um quadro de dor lombar crônica. Através de um estudo de revisão bibliográfica pretende-se entender o método, bem como informar sobre a eficiência do tratamento em pessoas que sofrem com a dor lombar crônica já que o Pilates demonstra proporcionar flexibilidade e estabilidade na musculatura da coluna, levando a uma melhora significativa na dor lombar, e consequentemente, na melhora da qualidade de vida.

Palavras-chave: Pilates; Dor Lombar Crônica; Qualidade de Vida. 


\begin{abstract}
Low back pain is commonly defined as pain or discomfort located in the area of the posterior aspect of the body, from the lower margin of the twelfth rib to the lower gluteal folds, with or without referred pain in one or both lower limbs. Chronic low back pain is characterized as a clinical condition of pain that persists for a period longer than three months. The Pilates Method presents itself as a clinical treatment that involves a physical and mental training program, which considers the body and the mind as a unit, thus being able to bring an overall improvement in the quality of life of individuals who present with chronic low back pain. Through a study of bibliographic revision it is intended to understand the method, as well as to inform about the efficiency of the treatment in people who suffer with chronic low back pain since Pilates demonstrates providing flexibility and stability in the spine musculature, leading to a significant improvement in low back pain, and consequently, in improving the quality of life.
\end{abstract}

Keywords: Pilates; Chronic Low Back pain; Quality of life.

\title{
1. Introdução
}

De acordo com Nascimento e Costa (2015), dor lombar é o conjunto de manifestações dolorosas que acometem a região inferior da coluna vertebral, podendo se manifestar de forma aguda ou crônica. A dor lombar crônica pode ser menos intensa, porém, é mais persistente e pode durar por mais de 3 meses até uma vida toda. Embora mais comum acima dos 50 anos, pode ocorrer em todas as idades. Ela é multifatorial e costuma indicar um problema bem mais grave na coluna vertebral. Normalmente, há queixas de travamento da coluna, limitação na flexão anterior da coluna, dor e limitações nos primeiros movimentos pela manhã.

Segundo Castellini et al. (2019), a dor lombar é comumente definida como dor ou desconforto localizado na área do aspecto posterior do corpo, desde a margem inferior da décima segunda costela até a pregas glúteas inferiores, com ou sem dor referida um ou ambos os membros inferiores. 
A dor lombar é um dos problemas de saúde mais comuns na população mundial, entretanto, ainda não existe um consenso na comunidade médica para caracterizar seus sintomas sendo, portanto, difícil categorizar esses pacientes dentro de grupos homogêneos (MACEDO, 2017).

O método Pilates foi desenvolvido pelo alemão Joseph Pilates, na década de 1920, e tem como base o conceito de contrologia. Esse termo designa o controle consciente de todos os movimentos musculares do corpo. O Pilates é um programa de treinamento físico e mental que considera o corpo e a mente uma unidade. O método está no fortalecimento do centro de força, do tronco inferior e da pelve, ativando principalmente os músculos, multífidos, assoalho pélvico e transverso abdominal, estrutura que suporta e reforça o tronco. A ativação correta desse centro de forças auxilia na postura, facilitando a realização de movimentos equilibrados do corpo com um todo. Os conceitos do método estão contidos na aplicação dos seis princípios básicos, fundamentais do Pilates: centro, concentração, controle, precisão, respiração e fluidez (SILVESTRE, 2015).

Este estudo trata-se de uma revisão bibliográfica, de caráter descritivo e exploratório, onde os critérios de inclusão das referências no estudo foram: artigos científicos que estavam nas bases de dados internacionais e nacionais, como GOOGLE ACADÊMICO, MEDLINE via PUBMED, LILACS, BIREME e PEDRO onde foi realizado e avaliado a qualidade metodológica dos estudos indexados com score de 8 a 10, um aprofundamento sobre o Método Pilates na dor lombar crônica. Foram consultados artigos entre os anos de 2011 a 2019, que falavam sobre dor lombar e Pilates na população do sexo feminino e masculino em várias faixas etárias. Gestantes com lombalgias utilizando o método Pilates como tratamento foram excluídas do estudo. Os artigos foram analisados por ordem cronológica de publicação, podendo assim averiguar a eficiência do tratamento mesmo com o passar dos anos.

O objetivo do estudo foi verificar a eficiência e os resultados obtidos em estudos que utilizaram o método Pilates como tratamento em pacientes com dor lombar crônica, além de pesquisar e estudar artigos que trouxeram informações e a utilização do método como um recurso para o tratamento das dores lombares. Justifica-se este estudo já que a dor lombar crônica é umas das disfunções musculoesqueléticas mais frequentes na atualidade, podendo levar à incapacidade funcional, afetando assim a qualidade de vida dos indivíduos acometidos. 


\section{Revisão bibliográfica}

\subsection{Princípios e benefícios do Pilates}

O método Pilates apresenta-se como um método de reabilitação e habilitação, fortalecendo as musculaturas que permitem estabilizar a coluna lombar entre eles estão os músculos multífidos e transverso do abdômen trazendo uma gama de benefícios como aumento da flexibilidade, tonificando a musculatura, alonga o corpo e um maior condicionamento físico (YAMATO et al., 2016).

Durante as sessões do Pilates voltadas para a dor lombar os exercícios são focados no fortalecimento abdominal e paravertebral, na mobilidade e na flexibilidade da coluna, e em exercícios para o corpo todo. Conforme o indivíduo vai progredindo nas execuções dos exercícios e os controles do quadro álgico são gradualmente introduzidos, exercícios de extensão do tronco, além de outros exercícios para o corpo todo, procuram melhorar a relação de equilíbrio agonista-antagonista (PEREIRA; CASA JUNIOR; CAMPOS, 2013).

É importante ressaltar que antes de realizar qualquer exercício do método Pilates é preciso aprender a respirar corretamente, realizando a inalação e exalação completa do ar. Sendo possível estimular os músculos a uma maior atividade, carregando oxigênio puro e promovendo melhor estado de saúde. Essa ênfase na respiração durante o método ajuda a nutrir o corpo e eliminar toxinas, melhorando a organização do tronco pelo recrutamento dos músculos estabilizadores profundos da coluna na sustentação pélvica, bem como o relaxamento dos músculos inspiratórios e cervicais (FERNANDES; LACIO, 2011).

A centralização é considerada o foco principal do método, que ocorre através da ativação dos músculos centrais do corpo, a concentração é fundamental para que corpo e mente, fique em sintonia. Quando o exercício se inicia do centro de força com concentração, é o controle do corpo que o praticante deve possuir e realizar os movimentos com cuidado. Outro princípio é a precisão, onde os movimentos devem ser sincronizados e harmônicos. Todos os exercícios devem ser realizados com ritmo, e por isso é importante a adequada 
respiração para que aconteça a melhor circulação de sangue oxigenado. E o último princípio é a fluidez, onde os exercícios fluem de um para o outro de forma suave e lenta (SILVA, 2017).

Os exercícios realizados possuem contrações isotônicas (concêntricas e excêntricas), mas principalmente isométricas, tendo como principal objetivo a contração do centro do corpo, chamado de powerhouse (casa de força). Esta casa de força é constituída pelos músculos abdominais, músculos do assoalho pélvico, glúteos e paravertebrais. Através da expiração juntamente com a contração dessas musculaturas e do diafragma é possível ter uma maior estabilização dinâmica e estática do corpo. A função do powerhouse é a estabilização da pelve e a da coluna durante o exercício, o adequado alinhamento da coluna, propiciar uma base para os movimentos, criar movimentos eficientes, gerar força para os movimentos e a prevenção de lesões. Os benefícios do treinamento do powerhouse são: equilíbrio corporal, melhora a estabilidade, a força postural aumenta e assegura o funcionamento adequado da cadeia cinética por completo (MARÉS, 2017).

Os benefícios do Pilates são inúmeros, desde que a execução dos exercícios seja realizada de forma fiel aos seus princípios. Algumas vantagens são: a estimulação da circulação; melhora do condicionamento físico; melhora da flexibilidade e mobilidade articular; melhora da postura; ganho de consciência corporal; melhora da coordenação motora e equilíbrio; melhora a capacidade respiratória; aumento da resistência e força muscular; ganho de consciência corporal. Tais benefícios ajudam a prevenir lesões e proporciona a redução da fadiga, desconforto e dor (COMUNELLO, 2011)

A mobilidade é gerada com a interação entre a função muscular e os sistemas de alavancas articulares sob o controle do sistema nervoso. Os músculos são compostos por tecidos contrateis e não contrateis, gerando resistência a forças deformantes. A extensibilidade é a capacidade de ceder e relaxar a uma força de alongamento que as unidades musculotendíneas que cruzam uma articulação possuem. Após o alongamento, o tecido mole tem a habilidade de retornar ao seu comprimento normal caracterizando a elasticidade (LEITÃO, SILVA, RASIA, 2013).

Entre os inúmeros métodos de treinamento, o Pilates é uma prática de exercícios físicos que combina o treino de força e flexibilidade. Para obter-se um bom programa para flexibilidade, primeiramente são planejados exercícios através de uma avaliação, que deveram 
ser realizados de forma regular. Os exercícios podem ser aumentados de forma progressiva a amplitude de movimento conveniente à capacidade do indivíduo (JUNGES, 2016).

Uma revisão de literatura realizada por Vieira e Fleck (2013), a respeito da influência do método Pilates na dor lombar crônica confirma os achados do estudo que relata que o método Pilates tem influência positiva no tratamento da lombalgia, melhorando a qualidade de vida, flexibilidade, consciência corporal, força muscular, propriocepção e correção postural.

\subsection{Efeitos do Pilates na dor lombar crônica}

O Pilates caracteriza-se por um conjunto de movimentos onde a posição neutra da coluna vertebral é sempre respeitada, objetivando a melhora da coordenação da respiração com o movimento do corpo, a flexibilidade geral, a força muscular e a postura, sendo, portanto, esses fatores essenciais no processo de reabilitação postural. Na prática desses exercícios musculares de baixo impacto, recomenda-se a utilização de seis princípios imprescindíveis: concentração, controle, precisão, fluidez do movimento, respiração e utilização do centro de força (COSTA, ROTH, NORONHA, 2012).

As aulas do método são realizadas de forma dinâmica por educadores físicos ou fisioterapeutas, com exercícios que englobam contrações musculares isotônicas e isométricas. Logo nas primeiras aulas, alguns benefícios podem ser notados, como melhora da mobilidade, flexibilidade e da força muscular; melhora da consciência corporal e coordenação motora; melhora também da postura, do equilíbrio e da respiração. O método pode ser praticado por todas as pessoas de diferentes idades (FARIA; FARIA, 2013).

Wajswelner, Metcalf e Bennell (2012) fizeram um estudo onde o objetivo era comparar a eficácia do Pilates na Fisioterapia com exercícios gerais para dor lombar crônica. O estudo foi feito de maneira experimental e participaram 87 voluntários, que foram divididos em dois grupos. O grupo dos exercícios gerais participou de duas sessões semanais de uma hora durante seis semanas. Já o grupo do Pilates recebeu um programa de exercícios específicos. Com isso, foi possível concluir que programas de exercícios clínicos específicos de Pilates são tão eficazes na redução da dor e incapacidade e na melhoria da função em 
adultos com dor lombar crônica quanto nos exercícios gerais tradicionais quando ambos os programas são usados por fisioterapeutas.

Em um estudo similar, Mostagi et al. (2015) concluíram que a dor lombar (DL) é uma das causas mais comuns de incapacidade, e o método Pilates tem sido associado a melhorias nos sintomas. Sendo assim, os autores se propuseram avaliar a eficácia do método Pilates, quando comparado aos exercícios gerais, sobre dor e funcionalidade após oito semanas, realizando 16 sessões em duas vezes por semana e acompanhamento de três meses, em indivíduos com não lombalgia crônica específica. Os indivíduos foram divididos em dois grupos: o grupo Pilates (GP) e o grupo geral de exercícios (GGE). O protocolo GP foi baseado no método Pilates e o GGE realizou exercícios para gerenciar a dor lombar crônica. Após o exposto concluíram que não houve diferenças entre os grupos. Quando se analisa ao longo do tempo, o GGE demonstrou melhorias na funcionalidade entre a linha de base e o final do tratamento $(\mathrm{P}=0,02)$. Percebeu-se que não houve diferenças entre o Pilates e os exercícios gerais em relação à dor e à funcionalidade em indivíduos com dor lombar crônica, mas os exercícios gerais foram melhores que o Pilates para aumentar a funcionalidade e a flexibilidade.

Stolze, Allison e Childs (2012) buscaram em sua pesquisa, derivar uma regra de predição clínica para identificar um subgrupo de pacientes com dor lombar crônica que provavelmente se beneficiariam do exercício baseado em Pilates. O Estudo contava com um grupo de 96 pacientes, onde 95 terminaram o estudo e sendo assim, dos 95 pacientes participantes, 51 obtiveram resultado positivo. A regra com 5 variáveis utilizando: amplitude de movimento total de flexão do tronco igual ou inferior a $70^{\circ}$, duração dos sintomas atuais de 6 meses ou menos, sem sintomas nas pernas na última semana, índice de massa corporal igual ou superior a $25 \mathrm{~kg} / \mathrm{m}^{2}$, e amplitude de movimento de rotação média do quadril esquerdo ou direito de $25^{\circ}$ ou mais. Se 3 ou mais dos 5 atributos estivessem presentes (probabilidade positiva, 10,64) a probabilidade de obter um resultado positivo aumentou de $54 \%$ para $93 \%$, concluindo que esta regra pode ser útil para determinar quais os pacientes poderiam ser beneficiados com o Pilates, se posteriormente validado.

Marshall et al. (2013) compararam um estudo publicado anteriormente com o efeito de 8 semanas de exercícios específicos do tronco e ciclismo estacionário nas medidas de resultados de crenças catastrofizantes e de prevenção do medo (questionário FABQ) em 
pacientes com lombalgia crônica inespecífica. Participaram desse estudo 64 indivíduos designados para 8 semanas de grupo específico de exercício de tronco (SEG) e o grupo de ciclismo estacionário (CEG). Após as 8 semanas, a incapacidade foi significativamente menor no grupo específico de exercício de tronco em comparação com o grupo de ciclismo estacionário $(\mathrm{d}=0,62, \mathrm{P}=0,018)$. A dor foi reduzida desde o início nos dois grupos após o treinamento $(\mathrm{P}<0,05)$, mas foi menor no grupo específico de exercício de tronco $(\mathrm{P}$ $<0,05)$. Os escores da FABQ foram reduzidos no SEG em 8 semanas e no CEG em 6 meses. Um programa supervisionado de oito semanas de Pilates, em grupo, teve melhores resultados estatístico de curto, mas não de longo prazo, em comparação com o ciclismo estacionário. $\mathrm{O}$ exame clínico para os participantes que aderiram sugere que não há diferença entre os grupos. Se um paciente com dor lombar adere a exercícios específicos do tronco ou ao ciclismo estacionário, é possível pensar que melhorias semelhantes podem ser alcançadas.

Em 2014, Luz et al. objetivaram comparar a eficácia do Pilates solo e Pilates baseado em equipamentos em pacientes com lombalgia crônica inespecífica, onde participaram 86 pacientes com dor lombar crônica inespecífica. Os pacientes foram alocados aleatoriamente em 1 de 2 grupos: um grupo de Pilates solo $(n=43)$ e um grupo de Pilates com equipamento $(n=43)$. Os participantes de ambos os grupos participaram de 12 sessões de Pilates por um período de 6 semanas. Os desfechos primários foram intensidade da dor e incapacidade. Os desfechos secundários foram efeito percebido global, incapacidade específica do paciente e cinesiofobia. Um avaliador cego avaliou os resultados na linha de base e seis semanas e seis meses após a randomização. Assim, concluíram que o Pilates baseado em equipamento foi superior ao Pilates solo no acompanhamento de 6 meses para os resultados de incapacidade e cinesiofobia.

Natur et al. (2015) avaliaram a eficácia do método Pilates em pacientes com lombalgia crônica inespecífica. O estudo foi realizado com 60 pacientes, divididos aleatoriamente em dois grupos: Grupo Experimental (GE) que manteve tratamento medicamentoso com uso de AINE e foi submetido a tratamento com o método Pilates e Grupo Controle (GC) que continuaram tratamento medicamentoso com uso de AINE e não foram submetidos a nenhuma outra intervenção. Assim, concluíram que o método Pilates pode ser utilizado por pacientes com lombalgia para melhorar a dor, função e aspectos 
relacionados à qualidade de vida (capacidade funcional, dor e vitalidade). Além disso, esse método não tem efeitos prejudiciais para esses pacientes.

A fim de investigar os efeitos de um programa de exercícios de Pilates na incapacidade, dor, mobilidade lombar, flexibilidade e equilíbrio em pacientes com lombalgia crônica inespecífica. Valenza et al. (2017) desenvolveram um estudo com 54 pacientes subdivididos em dois grupos aleatórios onde foram analisados: Incapacidade (Roland-Morris Disability Questionnaire e Oswestry Disability Index), dor atual, dor média e dor no seu pior ou mais doloroso período (Escalas Visual Analógicas), mobilidade lombar (teste de Schober modificado), flexibilidade (teste do dedo no chão) e equilíbrio (teste de postura de membro único) foram medidos no início e após a intervenção. Conseguiram demonstrar que entre os grupos houve diferenças significativas no grupo de intervenção em comparação com o grupo controle para os dois escores de incapacidade, o questionário Rolland-Morris e Índice de Incapacidade de Oswestry ( $p<0,001)$, dor atual $(p=0,002)$ e dor no mínimo $(p=0,033)$, flexibilidade $(0,032)$ e equilíbrio $(0,043)$. Por fim, conseguiram concluir que um programa de exercícios de 8 semanas de Pilates foi eficaz para melhorar a incapacidade, dor, flexibilidade e equilíbrio em pacientes com lombalgia crônica inespecífica.

No estudo de Bhadauria e Gurudut (2017), o objetivo do estudo foi comparar três formas diferentes de exercícios: estabilização lombar, fortalecimento dinâmico e Pilates na dor lombar crônica (dor lombar crônica) em termos de dor, amplitude de movimento, e força de tronco. Neste estudo, 44 indivíduos que sofrem de dor lombar inespecífica por mais de três meses foram alocados aleatoriamente no grupo de estabilização lombar, no grupo de fortalecimento dinâmico e no grupo Pilates. Foram prescritas dez sessões de exercícios por três semanas, juntamente com corrente interferencial e bolsa úmida quente. A dor foi avaliada pela escala visual analógica, incapacidade funcional pelo Oswestry Disability Questionnaire modificado, amplitude de movimento avaliando a flexão e extensão lombar pelo teste de Schober modificado e a força de tronco foi avaliado pelo biofeedback de tensão no $1^{\circ}$ e no $10^{\circ}$ dia do tratamento. Houve redução da dor, melhora na amplitude de movimento, capacidade funcional e centro de força em todos os três grupos de exercícios. A melhora foi significativamente maior no grupo de estabilização lombar para todas as medidas de desfecho, quando comparado ao pós-tratamento após a $10^{\mathrm{a}}$ sessão. A comparação pareada mostrou que houve maior redução da incapacidade no grupo Pilates do que no grupo de fortalecimento 
dinâmico. Concluiu-se que a estabilização lombar foi superior quando comparada ao fortalecimento dinâmico e ao Pilates na lombalgia inespecífica crônica. No entanto, os benefícios à longo prazo, precisam ser avaliados e comparados com estudos prospectivos de acompanhamento.

Cruz-Díaz et al. (2015) fizeram um estudo com 64 indivíduos, onde foram alocados aleatoriamente ao grupo de intervenção que consistiu na intervenção de Pilates durante 12 semanas ou no grupo controle que não recebeu tratamento. Foi possível verificar que houve diferenças significativas entre os grupos com melhora observada no grupo de intervenção Pilates em todas as variáveis após o tratamento $(\mathrm{P}<0,001)$. Grandes alterações na incapacidade e cinesiofobia foram observadas em seis semanas de intervenção, sem diferença significativa após 12 semanas $(\mathrm{P}<0,001)$. As alterações médias do grupo intervenção em comparação com o grupo controle foram de 4,00 $(0,45)$ no questionário de Incapacidade Roland Morris e de 5,50 (0,67) na Escala Tampa de Cinesiofobia. A dor mostrou melhores resultados em seis semanas, com uma melhora leve, mas estatisticamente significativa, em 12 semanas, com escores da Escala Visual Analógica ( $P<0,001)$. Sendo assim, foi possível concluir que a intervenção do Pilates em pacientes com dor lombar crônica inespecífica foi eficaz no tratamento da incapacidade, dor e cinesiofobia.

Seguindo ainda a linha de raciocínio dos estudos já analisados, Miyamoto et al. (2018) apresentaram uma pesquisa com 296 indivíduos alocados em quatro grupos, onde cada grupo recebeu uma orientação: grupo de folhetos, Pilates uma vez por semana, Pilates duas vezes por semana e Pilates três vezes por semana. Com isso, foi possível averiguar que a adição de duas sessões de exercícios de Pilates ao aconselhamento proporcionou melhores resultados em dor e incapacidade do que o aconselhamento isolado para pacientes com dor lombar crônica; elementos inespecíficos, como maior atenção ou expectativa, podem fazer parte desse efeito. A análise de custo-utilidade mostrou que o Pilates três vezes por semana era a opção preferida.

\section{Considerações finais}


O método Pilates pode ser utilizado por fisioterapeutas para diversos fins e no tratamento da dor lombar crônica. $\mathrm{O}$ método se apresenta como uma ferramenta importante para diminuir e/ou dissipar a dor.

Através das evidências encontradas nesta revisão, o método Pilates foi considerado eficaz para a diminuição da dor, além de melhorar o quadro de incapacidade comumente observado por indivíduos com dor lombar crônica, pois o método trabalha o ganho de força, flexibilidade, equilíbrio e estabilização da coluna lombar. Porém, na maioria dos estudos o método Pilates não foi superior quando comparado aos exercícios gerais.

Baseado nesses achados, é possível concluir que a abordagem fisioterapêutica juntamente com o método Pilates demonstrou-se eficaz no tratamento da dor lombar crônica contribuindo de forma positiva na percepção da qualidade de vida, por ser um método que não tem contraindicações a sua intervenção pode na maioria das vezes proporcionar maiores ganhos comparados ao grupo controle.

Sugere-se que mais estudos com número maior de pacientes sejam realizados com o Método Pilates, especialmente no que se refere aos resultados à longo prazo.

\section{Referências}

BHADAURIA, E A; GURUDUT P. Comparative effectiveness of lumbar stabilization, dynamic strengthening, and Pilates on chronic low back pain: randomized clinical trial. J Exerc Rehabil. 2017 Aug 29;13(4):477-485.

CASTELLINI, G; GIANOLA, S; ANDREANO, A; CORBETTA, D; FRIGERIO P; PECORARO, V; REDAELLI, V; TETTAMANTI, A; TUROLLA, A; MOJA, L; VALSECCHI M G. Eficácia dos tratamentos para doenças agudas e baixo subespecífico mecânico inespecífico dor nas costas: protocolo para revisão sistemática e meta-análise de rede. (2019) 8: 196.

COMUNELLO, J. F. Método Pilates: Aspectos históricos e princípios norteadores: uma revisão de literatura. Instituto Salus, maio-junho 2011, Passo Fundo/RS. Disponível em: $<$ http://scholar.google.com.br/scholar?q=historia+do+pilates\&hl=ptEN\&as_sdt=0\&as_vis=1 $\&$ oi=scholartsa $=$ X\&ei=dN7KVMKHHMihNvSUhJgM\&sqi $=\quad 2 \& v e d=0 C B 0 Q g Q M W A A>$. Acesso em 22/11/2019. 
CRUZ-DÍAZ D, MARTÍNEZ-AMAT A, DE LA TORRE-CRUZ MJ, CASUSO RA, DE GUEVARA NML, HITA-CONTRERAS F. Effects of a six-week pilates intervention on balance and fear of falling in women aged over 65 with chronic low-back pain: A randomized controlled trial. J.Maturitas. dez 2015, Volume 82, Issue 4, Pages 371-376

FERNANDES, L. V.; LACIO, M. L. O método Pilates: estudo revisional sobre seus benefícios na terceira idade. Revista Eletrônica da Faculdade Metodista Granbery. Juiz de Fora, n.10, 2011.

JUNGES, Silvana. Método Pilates - Paixão \&Ciência. AGE, p. 11, 2016.

KRAWCZKY B, MAINENTI MRM, PACHECO AGF. Efeito dos exercícios do método Pilates no alinhamento postural de adultos saudáveis. Rev Bras Med Esporte 2016; 22 (6): 485-90.

LEITÃO M.C; SILVA A.E.A; RASIA D. O método pilates aplicado em bailarinos clássicos para ganho de flexibilidade e força muscular, 2013. Disponível em: <http://200.230.184.11/ojs/index.php/CCBS/article/view/54/44>. Acesso em: 22/11/2019.

LUZ, M. A., COSTA, L. O. P., FUHRO, F. F., MANZONI, A. C. T., OLIVEIRA, N. T. B., \& CABRAL, C. M. N. (2014). Effectiveness of mat Pilates or equipment-based Pilates exercises in patients with chronic nonspecific low back pain: a randomized controlled trial. Physicaltherapy, 94 (5), 623-631.

MACEDO, Thuane Lopes; LAUX, Rafael Cunha; CORAZZA, Sara Teresinha. O efeito do método Pilates de solo na flexibilidade de idosas. Consciência e Saúde, v. 15, n. 3, p. 448456, 2017.

MARÉS, Gisele et al. A importância da estabilização central no método Pilates: uma revisão sistemática. Fisioterapia em movimento, v.25, n.2, 2017.

MARSHALL, Paul W. M., PhD; Kennedy, Suzanne, BHSc; Brooks, Cristy, BHSc; Lonsdale, Chris, PhD. Pilates exercise or stationary cycling for chronic nonspecific low back pain: does it matter? a randomized controlled trial with 6-month follow-up. Spine, July 01, 2013 Volume 38 - Issue 15 - p E952-E959

MIYAMOTO G C; FRANCO K F M; VAN DONGEN J M; FRANCO Y R D S; DE OLIVEIRA N T B; AMARAL D D V; BRANCO A N C; DA SILVA M L; VAN TULDER M W; CABRAL C M N. Different doses of Pilates-based exercise therapy for chronic low back pain: a randomised controlled trial with economic evaluation. Br J Sports Med. 2018 Jul; 52 (13): 859-868 
MOSTAGI, F Q; DIAS, J M; PEREIRA, L M; OBARA, K; MAZUQUIN, B F; SILVA, M F; SILVA, M A; DE CAMPOS R R; BARRETO, M S; NOGUEIRA, J F; LIMA, T B; CARREGARO R L; CARDOSO J R. Pilates versus general exercise effectiveness on pain and functionality in non-specific chronic low back pain subjects. J Body Mov Ther. 2015 Oct; 19 (4): 636-45.

NASCIMENTO, P. R. C.; COSTA, L. O. Prevalência da dor lombar no Brasil: uma revisão sistemática. Cad. Saúde Pública, Rio de Janeiro, v.31, n.6, p.1141-1155, jun. 2015.

NATUR J, CAZOTTI LDA, RIBEIRO LH, BAPTISTA AS, JONES A. Pilates improves pain, function and quality of life in patients with chronic low back pain: A randomized controlled trial. Clin. Rehabil. 2015; 29 (1): 59-68.

SILVA, Anne Caroline Luz Grudtner; MANNRICH, Giuliano. Pilates na reabilitação: Uma revisão sistemática. Fisioterapia em movimento, v. 22, n. 3, 2017.

SILVESTRE, N. O. P. Eficácia do método Pilates na dor lombar - estudo de revisão. 2015. 16f. Artigo científico apresentado ao curso de Especialização em Pilates do Centro de Estudos Avançados e Formação Integrada, chancelado pela Pontifícia Universidade Católica de Goiás (Especialização) - Pontifícia Universidade Católica (PUC), Goiás, 2015.

STOLZE L, ALLISON SC, CHILDS JD. Derivation of a preliminary clinical prediction rule for identifying a subgroup of patients with low back pain likely to benefit from pilates-based exercise.J Orthop Sports Physi. 2012; 42 (5): 425-36

VALENZA, M C; RODRÍGUEZ-TORRES, J; CABRERA-MARTOS I; DÍAZ-PELEGRINA A; AGUILAR-FERRÁNDIZ M E; CASTELLOTE-CABALLERO Y. Results of a Pilates exercise program in patients with chronic non-specific low back pain: a randomized controlled trial. Clin Rehabil. 2017 Jun; 31 (6): 753-760.

WAJSWELNER H, METCALF B, BENNELL K. Clinical Pilates versus general exercise for chronic low back pain: randomized trial. Med Sci Sports Exerc. 2012; 44 (7): 1197-205.

YAMATO TP, MAHER CG, SARAGIOTTO BT, HANCOCK MJ, OSTELO RW, CABRAL $\mathrm{CM}$ et al. Pilates for low back pain: complete republication of a cochrane review. Spine. 2016; 41 (12):1013-1021. 